\title{
Relationship between intra-operative hyperthermia, post-operative fever and local infectious complications in children undergoing cochlear implant surgery
}

\author{
Ziv Ribak ${ }^{1}$, Muhammad Abu Tailakh ${ }^{2}$, Mordechai Kraus ${ }^{1}$, Daniel KAPLAN ${ }^{3}$, and Sabri \\ El-Saied $^{1}$ \\ ${ }^{1}$ Soroka Medical Center \\ ${ }^{2}$ Ben-Gurion University of the Negev \\ ${ }^{3}$ Soroka Hospital
}

November 2, 2020

\begin{abstract}
Objective: To examine the relationship between Intra-operative hyperthermia (IOH) and post-operative fever (POF) and local complications in children undergoing CI surgery. Study Design: Retrospective cohort study Setting: Tertiary care University Hospital. Participants: The study includes all pediatric CI surgery procedures conducted in one hospital in Israel between 2007 and 2017, A total of 213 CI procedures were performed on 191 children (ages 9 months to 17 years; mean 3.54 years) Main outcome and measure: Clinical data included demographics, type of surgery (unilateral, bilateral), presence of IOH and POF, and local infectious complications within one month after surgery Results: Ten patients (4.9\%) developed IOH, of which two developed POF. Of the remaining non-IOH cases (95.1\%), 29 children (14.3\%) developed POF. IOH correlated with cases of bilateral CI $(80 \%$ bilateral CI versus $20 \%$ unilateral CI; p = 0.002). IOH also correlated with the duration of operation $(289$ min versus $189 \mathrm{~min}$, respectively; $\mathrm{p}=0.025)$. Local complications were recorded in 30 patients: two that developed POF in the $\mathrm{IOH}$ group, and $28(14.3 \%)$ in the non-IOH group. No correlation was observed between the occurrence of either IOH or POF, and the occurrence of local complications. Conclusions: IOH and POF are not uncommon in children undergoing CI surgery, yet, in the present study cohort, both conditions are not associated with the development of local infectious complications. In addition, IOH does not appear to predict the development of POF.
\end{abstract}

Relationship betweenintra-operative hyperthermia, post-operative fever and local infectious complications in children undergoing cochlear implant surgery

Running title: Intra-operative hyperthermia ( $\mathrm{IOH})$ and post-operative fever (POF) during CI surgery

Conflicts of interest: None

Financial disclosures: None

Availability statement: The data that support the findings of this study are available from the corresponding author, upon reasonable request.

\section{Abstract}

Objective: To examine the relationship between Intra-operative hyperthermia (IOH) and post-operative fever (POF) and local complications in children undergoing CI surgery.

Study Design: Retrospective cohort study 
Setting: Tertiary care University Hospital.

Participants: The study includes all pediatric CI surgery procedures conducted in one hospital in [Blinded for review] between 2007 and 2017, A total of 213 CI procedures were performed on 191 children (ages 9 months to 17 years; mean 3.54 years)

Main outcome and measure: Clinical data included demographics, type of surgery (unilateral, bilateral), presence of $\mathrm{IOH}$ and $\mathrm{POF}$, and local infectious complications within one month after surgery

Results: Ten patients (4.9\%) developed IOH, of which two developed POF. Of the remaining non-IOH cases (95.1\%), 29 children (14.3\%) developed POF. IOH correlated with cases of bilateral CI ( $80 \%$ bilateral CI versus $20 \%$ unilateral $\mathrm{CI} ; p=0.002$ ). IOH also correlated with the duration of operation (289 min versus 189 min, respectively; $p=0.025$ ). Local complications were recorded in 30 patients: two that developed $\mathrm{POF}$ in the IOH group, and $28(14.3 \%)$ in the non-IOH group. No correlation was observed between the occurrence of either IOH or POF, and the occurrence of local complications.

Conclusions: IOH and POF are not uncommon in children undergoing CI surgery, yet, in the present study cohort, both conditions are not associated with the development of local infectious complications. In addition, IOH does not appear to predict the development of POF.

Keywords : Anesthesia, deafness, pediatric population, susceptibility to infection, complications.

\section{Key points}

- Post-operative fever and intra-operative hyperthermia are commonly encountered in all surgical disciplines

- The role of both entities in children undergoing cochlear implant is unclear

- Its relationship and the effect on local complications is presently unknown

- Here, IOH and EPF are found to be not uncommon in children undergoing CI surgery.

- Both seem to be benign conditions and do not influence the development of local infectious complications

\section{Introduction}

Cochlear implantation (CI) is a well-defined and particularly safe surgical procedure that allows rehabilitation of hearing loss in patients with severe to profound sensorineural hearing loss. Nonetheless, surgical complications might include meningitis, wound infection, CSF leaks and facial paralysis [1-3].

The phenomenon of pediatric intra-operative hyperthermia (IOH) is well known; its occurrence in children undergoing CI has recently been described [4]. While the etiology of $\mathrm{IOH}$ remains unknown, it is suggested to follow a genetic predisposition or, in the particular case of CI surgery, the disbalance of thermoregulation by hypothalamic disruption [4-5].

Post-operative fever (POF) is also a common condition following surgical procedures [7-8]. While it rarely indicates an infection or other complication, it often leads to costly fever workups. We have previously described early POF after pediatric CI surgery (up to 72 hours after surgery) with an incidence of $19.2 \%$ [9]. It was unrelated to local infectious complications and long-term implications.

In the present study, we examine the relationship between IOH and POF in children who underwent CI surgery, and their relation to post-operative local infectious complications.

\section{Materials and methods}

Retrospective cohort study

\section{Ethical considerations}

The Institutional Ethics Committee approved the study.

\section{Participants}


All cases of children younger than 18 years who underwent CI surgery at the [Blinded for review] between 2007 and 2017 were included. Collected data included age, sex, type of surgery (unilateral, bilateral), event of intra-operative hyperthermia, post-operative fever, and the occurrence of local infectious complications. Body temperature was determined at the beginning of the operation, during the operation and at the end of the operation. For each patient, data included intravenous agents and the length of operation.

\section{Intra-operative hyperthermia and post-operative fever}

IOH during CI surgery and POF within the first 72 hours after CI surgery, were defined as an elevation in body core temperature of $38^{\circ} \mathrm{C}$ or above, irrespective of site of measurement (i.e., nasopharynx or rectum). Same-side post-operative local infectious complications were defined as any local swelling, redness, abscess, acute otitis media, acute mastoiditis or acute meningitis within one month after surgery.

All patients received intra-operative IV antibiotic prophylaxis (amoxicillin-clavulanic acid, $25 \mathrm{mg} / \mathrm{kg}$ ) and continued with two more doses along the first 24 hours.

Statistical analyses

Patient characteristics and results are reported as means \pm standard deviations for normally distributed quantitative data or as medians and interquartile ranges for non-normally distributed data. Nominal and categorical variables are expressed as numbers (percentages). The primary outcomes were post-operative complications (yes/no) and POF (yes/no). A Chi-square test was used for dichotomous variables and a Student's t-test was used for continuous variables. Multivariate binary logistic regression was used to evaluate independent association between patient characteristics and the development of complications or POF as a dichotomous variable.

All statistical analyses were performed using IBM SPSS Statistics, version 25. Results were considered statistically significant at $\mathrm{p}<0.05$.

Results

Demographics

Between the years 2007 and 2017, 191 patients aged 9 months to 17 years (mean 3.54 years), underwent 213 procedures, accounting for 260 cochlear implants at SUMC. Of these, 69 procedures (32\%) were simultaneous bilateral cochlear implants. Etiology was y hereditary in $80 \%$; two patients were operated for post-meningitis deafness; four procedures were for inner-ear congenital malformations ( 2 for incomplete partition type and 2 with an enlarged vestibular aqueduct). Of all procedures, 88 were performed on males (41\%) and 125 on females (59\%). One hundred procedures were performed on Bedouin Arab patients (63\%) and 72 on no Bedouin (usually Jewish) patients (34\%). Demographic and clinical characteristics are summarized inTable 1

Patients with IOHversus patients without IOH

Out of 213 procedures, 10 patients developed IOH and the rest were defined as belonging to the non-intraoperative hyperthermia (NIOH) group. No significant differences were observed between the groups in as far as age, gender, ethnicity, number of CI surgeries (one versus more than one), or the cause of deafness (Table 1 ).

\section{IOH versus length of surgery}

One hundred and forty-four patients underwent unilateral CI, 69 patients that underwent bilateral CI. There was no difference regarding the etiologies of deafness between unilateral and bilateral procedures. IOH was observed in 8 patients in the bilateral CI group (80\%) and two in the unilateral CI group (20\%) $(\mathrm{p}<0.05$,Table 1$)$.

IOH versus $P O F$ 
POF was observed following 31 procedures. Of these, 2 were from the 10-patient IOH group; no infections were found that could explain the fever and no antibiotic treatment was initiated in those patients. Neither of these two patients suffered from early post-operative complications. The rest of the patients with POF (29 patients) were in the NIOH group (14.3\%). In four patients there was a clear etiology for the POF (pharyngitis, gastroenteritis, periorbital cellulitis or dysentery). Five of the 29 patients showed post-operative complications, which included swelling (three patients), otitis media (one patient), and acute bacterial meningitis (one patient). According to multivariate analysis, IOH during CI surgery is not associated with the occurrence of POF ( $\mathrm{p}=0.923$; Table 2$)$.

\section{Post-operative local complications}

There was no significant difference in post-operative complications between the IOH and NIOH groups. Specifically, post-operative inflammatory complications were observed in two patients of the IOH group (one with local swelling and one with acute otitis media) and in 28 patients of the NIOH group (14.3\%; thirteen with swelling, eight with otitis media, four with local infection, two with meningitis, and one with otitis externa). According to multivariate analysis, $\mathrm{IOH}$ is not associated with post-operative complications $(\mathrm{p}=$ 0.89, Table 3 ).

\section{Intra-operative parameters}

Intra-operative data (i.e., duration of operation, minute-by-minute temperature recordings, intravenous agents employed, intra-operation complications and ICU admission) were available for 108 patients (Table 1 ). According to these, the duration of anesthesia was significantly longer in the IOH group compared to the NIOH group (289 min and 189 min, respectively). One of the patients in the IOH group was admitted to the ICU with suspected malignant hyperthermia and his operation was stopped after 182 minutes.

There was no significant difference in temperatures at the beginning of the operations between the IOH and $\mathrm{NIOH}$ groups $\left(35.9^{\circ} \mathrm{C}\right.$ and $35.8^{\circ} \mathrm{C}$, respectively; $\left.\mathrm{p}=0.639\right)$; however, statistical significance was observed for temperature at the end of the operation between the groups $\left(38.3^{\circ} \mathrm{C} \mathrm{IOH}\right.$ and $\left.36.7^{\circ} \mathrm{C} \mathrm{NIOH} ; \mathrm{p}<0.001\right)$. Peak temperature measured in the $\mathrm{IOH}$ group was $38.3^{\circ} \mathrm{C}$, and peak temperature measured in the NIOH group was $36.9^{\circ} \mathrm{C}(\mathrm{p}<0.001)$.

A positive and significant correlation was found between body temperature and duration of surgery $(\mathrm{p}<0.05)$, as well as between peak temperature during the operation and the duration of the operation $(\mathrm{p}<0.001)$ (Figure 1 ). Two patients were admitted to the ICU: one due to suspected malignant hyperthermia (IOH group) and the other due to low oxygen saturation during the operation (NIOH group).

Intravenous agents used during the operation included Propofol, Perfalgan, Neostigmine, Atropine, Midazolam, Emerone, Tracium and Morphine; no differences were evident in the use of these agents between the $\mathrm{IOH}$ and NIOH groups.

\section{Discussion}

The focus of this study was to examine the relation between IOH and POF in children undergoing CI surgery, and to explore their association with local complications. IOH has been previously described by Schwartz A et al. [4], who concluded that patients undergoing CI surgery are susceptible to IOH, without an identified etiology. There was, however, a higher incidence of IOH in CI surgeries compared to anatomically similar surgeries, such as mastoid or ophthalmic surgeries, as well as a significant difference in the length of anesthesia between $\mathrm{IOH}$ and $\mathrm{NIOH}$.

In the present study, IOH appears to be a benign phenomenon which does not predispose the patient to POF or to post-operative inflammatory complications, as per the multivariate analysis.

The overall incidence of IOH during CI in the present study $(4.7 \%)$ is lower than that which was reported by Schwartz A et al. (10\%), meaning that the phenomenon became less frequent in recent years. Interestingly, in the more recent cases, there was a larger fraction of bilateral implants, which is the group of patients that initially had a higher rate of $\mathrm{IOH}$. While a bilateral procedure is longer in duration, thus exposing the 
patient to prolonged anesthesia, intravenous anesthesia has been replacing inhaled anesthesia in the past years [4].

According to the present study, IOH is not associated with the occurrence of POF. Indeed, POF is not unusual in CI surgery; compared to an incidence of $19.2 \%$ [9], the incidence of POF in the present study $(14.5 \%)$ continues to represent a benign entity with no major clinical significance. However, the financial burden of a POF-related work up remains high.

The post-surgical complication rate in the present study was similar to the rate found in the literature [1]. However, while many studies examined complications after CI surgeries, the present study focused on inflammatory complications, with the rationale of a possible common axis that connects IOH, POF and infectious complications.

The present study has several limitations. Being a retrospective study, data on complications that had occurred after patient discharge may be lacking. In addition, sample size in the intra-operative analysis is relatively small: Out of 190 patient records, only 108 had sufficient data across study parameters. Therefore, a robust conclusion regarding a putative correlation between length of anesthesia and hyperthermic events could not be made. A larger study with a more detailed documentation of each parameter per patient would be required in order to learn how factors such as anesthesia, room temperature and heating methods, may affect hyperthermic events in CI surgery.

Taken together, based on the presently studied cohort, IOH and POF are not unusual in children undergoing CI surgery, and are independent of each other. Indeed, here, IOH does not predict the development of POF. Moreover, both hyperthermic events do not seem to influence the development of local complications.

\section{References}

[1] Bhatia K, Gibbin KP, Nikolopoulos TP, O'Donoghue GM. Surgical complications and their management in a series of 300 consecutive pediatric cochlear implantations. OTOLOGY \& NEUROTOLOGY. 2004;25:730-9.

[2] Daneshi A, Ajalloueyan M, Ghasemi MM, Hashemi BS, Emamjome H, Farhadi M, Ajalloueyan Z. Complications in a series of 4400 paediatric cochlear implantation. INTERNATIONAL JOURNAL OF PEDIATRIC OTORHINOLARYNGOLOGY 2015;79:1401-3.

[3] Arnoldner C, Baumgartner WD, Gstoettner W, Hamzavi J. Surgical considerations in cochlear implantation in children and adults: a review of 342 cases in Vienna. ACTA OTO-LARYNGOLOGICA. 2005;125:22834.

[4] Schwartz A, Kaplan D, Rosenzweig V, Klein M, Gruenbaum BF, Gruenbaum SE, et al. The incidence of hyperthermia during cochlear implant surgery in children. THE JOURNAL OF LARYNGOLOGY \& OTOLOGY 2017;15:1-7.

[5] Insler SR, Sessler DI. Perioperative thermoregulation and temperature monitoring. ANESTHESIOLOGY CLINICS OF NORTH AMERICA 2006;24:823-837.

[6] Merdad M, Crawford M, Gordon K, Papsin B. Unexplained fever after bilateral superficial cervical block in children undergoing cochlear implantation: an observational study. CANADIAN JOURNAL OF ANESTHESIA 2012 Jan;59:28-33.

[7] Guinn S, Castro FP, Jr, Garcia R, Barrack RL. Fever following total knee arthroplasty. THE AMERICAN JOURNAL OF KNEE SURGERY 1999;12:161-164.

[8] Perlino CA. POSTOPERATIVE FEVER. MEDICAL CLINICS OF NORTH AMERICA 2001;85:11411149 .

[9] El-Saied S, Joshua BZ, Abu Tailakh M, Jagev I, Gorali R, Kraus M, Kaplan DM. Early postoperative fever in pediatric patients undergoing cochlear implant surgery. CLINICAL OTOLARYNGOLOGY 2018;43:385388. 


\section{Hosted file}

Tab.COA.pdf available at https://authorea.com/users/372196/articles/490312-relationshipbetween-intra-operative-hyperthermia-post-operative-fever-and-local-infectiouscomplications-in-children-undergoing-cochlear-implant-surgery

\section{Hosted file}

Figure COA.pdf available at https://authorea.com/users/372196/articles/490312-relationshipbetween-intra-operative-hyperthermia-post-operative-fever-and-local-infectiouscomplications-in-children-undergoing-cochlear-implant-surgery 